\title{
Accuracy of High Resolution Multidetector Computed Tomography in the Local Staging of Rectal Cancer
}

\author{
Rochita Venkataramanan ${ }^{10}$ Venkatesh Munikrishnan² Akash Venkataramanan ${ }^{3}$ \\ Sudeepta Kumar Swain ${ }^{2}$ Kaveripattu Sathiyamurthy Sunilkumar ${ }^{4}$ Vadanika Venu ${ }^{1}$ \\ Muthuswamy Hariharan ${ }^{5}$ Mohammad Zehran Saipillai ${ }^{6} \quad$ Asfar Ahamed $^{2}$
}

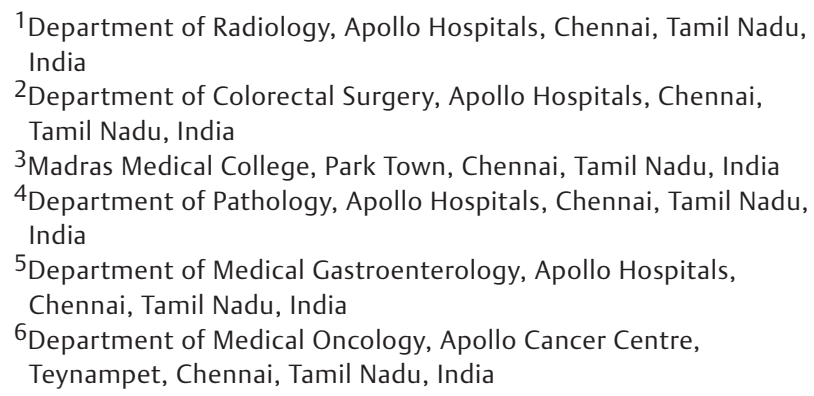

Address for correspondence Rochita Venkataramanan, MD, DNB, DMRD, No 34, Srinivasa Murthy Avenue, Off L B Road, Adyar, Chennai 600020, Tamil Nadu, India (e-mail: rochitav@yahoomail.com, rochitav@gmail.com).

J Gastrointestinal Abdominal Radiol ISGAR 2022;5:1-15

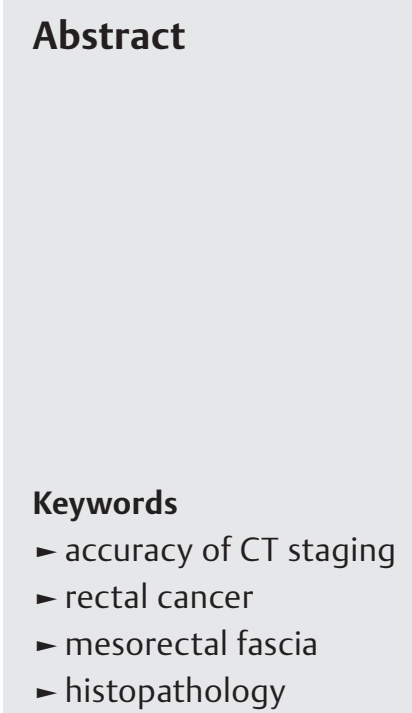

Background Magnetic resonance imaging (MRI) is the gold standard for local staging of rectal cancer. Advanced computed tomography (CT) machines are now capable of high-resolution images of rectal cancer and utilized for $\mathrm{CT}$ perfusion. The possibility of local staging of rectal cancer by $\mathrm{CT}$ needs to be explored.

Purpose The aim of the study is to evaluate accuracy of high-resolution CT for local rectal cancer staging.

Methods A high-resolution CT was performed for local staging of rectal cancer in our study group of 93 patients, where 64 underwent primary surgery and 29 underwent surgery post neoadjuvant chemoradiotherapy (NACRT).

Results In differentiating stages T2-and-less than T2 from T3-T4 rectal cancer, accuracy, sensitivity, specificity, and kappa score in overall patients were $91 \%, 87 \%$, $94 \%$, and 0.8 ; in primary surgery group were $89 \%, 76 \%, 94 \%$, and 0.7 ; in NACRT group were $97 \%, 100 \%, 94 \%$, and 0.9 ; in low rectal group were $94 \%, 89 \%, 97 \%$, and 0.82 , respectively.

Conclusion High resolution CT is an accurate tool for local staging of rectal cancer.

\section{Introduction}

Colorectal cancer is the second most common malignancy across the world. One-third of these cases are rectal cancers (RCs) with over $40 \%$ arising within $6 \mathrm{~cm}$ of the anal verge. ${ }^{1}$ Radiologic imaging modalities have assumed vital importance in the staging of these patients as there has been a paradigm shift in the management of RC. The adoption of

published online April 30, 2021
DOI https://doi.org/ $10.1055 / \mathrm{s}-0041-1726662$ ISSN 2581-9933 the technique of total mesorectal excision as well as neoadjuvant chemoradiotherapy (NACRT) prior to surgery in advanced RC has improved the chances of survival by dropping local recurrence rates remarkably. Additionally, better survival of metastatic $\mathrm{RC}$ has been demonstrated by resection of liver and lung secondaries. Accurate local staging of RC and the relationship of the disease to the mesorectal fascia (MRF) or the $\mathrm{T}$ stage as well as identification of metastatic nodes 
( $N$ stage), are the bases of choosing patients for primary surgery (PS) versus NACRT.

At the turn of the century, comparison of multidetector computed tomography (CT) and magnetic resonance imaging (MRI) by several authors showed conflicting reports with some papers suggesting CT to be superior to MRI in T1-T2 RC staging. ${ }^{2}$ Others mention that due to its inherent low contrast resolution, CT lacks sufficient accuracy for distinction between the individual bowel wall layers. ${ }^{3}$ Consequently, MRI has evolved as the standard for local staging of RC and CT for distant staging. However, recent meta-analysis suggests an equal accuracy of CT as compared with MRI for local staging. ${ }^{4}$ Some suggest accuracy not only for high and mid RC but even for low RC with better T2-staging than MRI. ${ }^{5} \mathrm{CT}$ perfusion, a recent application in $\mathrm{RC}$ is utilized to assess tumor biology and grading which affect survival as well as response to NACRT. The principle used is that due to a higher blood flow, blood volume, and vascular permeability the RC enhances differently from the normal mucosa. ${ }^{6}$ However, the guidelines for local RC staging continue to recommend MRI. Requirement of performing both MRI for local staging and CT for distant staging is expensive. Against this background we embarked on a prospective blinded observational study to evaluate whether CT could be utilized as a single modality to assess overall staging, local and distant for RC.

\section{Materials and Methods}

\section{Patient Population}

A prospective observational blinded study was performed from 2017 to 2019. The study group consisted of consecutive 93 patients, 69 men and 26 women with mean ages of 45 and 51 years, respectively. All patients were discussed at a multidisciplinary team meeting. Of these, 64 patients underwent PS, and 29 patients underwent surgery post NACRT. The NACRT course consisted of 5.5 weeks delivery of a dose of 45 to 50 Gray in fractions and a concomitant chemotherapy with capecitabine or 5-fluorouracil. Surgery was performed at an interval of 6 to12 weeks after completion of chemoradiation. The number of patients with high, mid, and low RC were 22, 29 , and 42, respectively. Informed consent from all patients and Institutional Ethics Committee approval were obtained.

\section{High-Resolution CT Protocol}

All patients underwent a mild bowel preparation with $1 \mathrm{~L}$ of polyethylene glycol the night before the examination. Oral or rectal positive contrast was not administered as this interferes with the evaluation of the mucosa. ${ }^{7} \mathrm{CT}$ was performed on Aquilion One CT (Canon, Tokyo, Japan) in the 64-detector mode with intravenous contrast of 1 to $1.5 \mathrm{~mL} / \mathrm{kg}$ iopromide (Ultravist 370; Schering, Berlin, Germany) at the rate of $4 \mathrm{~mL} / \mathrm{s}$. Scans were obtained during the arterial, early venous phase (EVP) of 40 seconds and a later venous phase (LVP) of 70 seconds delay after contrast injection with a $0.5-\mathrm{mm}$ section thickness. The EVP gave the maximum contrast between the rectal wall and the RC. The arterial scan was included for vascular mapping needed for the resection of hepatic metastases as per institutional protocol. We improved the scan resolution of the EVP by reducing the field-of-view (FOV). ${ }^{8,9}$
The combination of factors that yielded one significantly high-resolution CT phase were:

1. Inclusion of an EVP at 40 seconds from injection.

2. Sixty-four detector rows, $0.5-\mathrm{mm}$ collimation with a pitch factor of 0.8 .

3. Mild bowel cleansing 8 hours prior to scan.

4. 100 to $130 \mathrm{~mL}$ of 350 to $370 \mathrm{mg} / \mathrm{mL}$ of iodinated intravenous contrast at $4 \mathrm{~mL} / \mathrm{s}$.

5. Reducing the FOV to include up to the bony pelvic side walls laterally and from the sigmoid colon to the perineum craniocaudally, typically approximately $16 \mathrm{~cm}$.

\section{Image Analysis}

The images were analyzed on a dedicated Vitrea workstation (Vital Images, Minnetonka, Minnesota, United States). Multiplanar reconstructions (MPRs) were performed and read by a radiologist with 26 years of experience and another with 7 years of experience with decision by consensus. Oblique reconstructions were used to obtain a "true axial plane" orthogonal to the anorectum (-Fig. 1A-D). Curvilinear reconstructions (CLR) were done with the centerline through the lumen of the rectum. These yielded true axial sections as well as a long axis straightened-out view bringing the anorectum in one two-dimensional (2D) image that can be rotated 360 degrees ( - Fig. 1E, F). Depth of transmural invasion was categorized as ctT1, ctT2, ctT3, and ctT4, according to the AJCC TNM classification.

In the EVP, the rectal mucosa was seen as a brightly enhancing line as compared to the muscularis propria (MP). The submucosa was seen as a hypodense layer. The MP and internal sphincter(IS) are hypodense to the mucosa and isodense to the gluteal muscles. The RC enhanced as much as the mucosa or slightly more or less on this phase and was well differentiated from the MP ( - Fig. 2). Mucinous tumors had fluid density and enhanced far less than the mucosa as well as the MP ( $\mathbf{F i g}$. $3 \mathbf{A}, \mathbf{B}$ ).

The MRF was identified as a thin line around the mesorectum (-Fig. 2C). MRF was considered involved in all T4 RC ( - Fig. 3C-F). and in T3 RC when the distance between the tumor margin and MRF was $<1 \mathrm{~mm}$.

Extramural vascular invasion (EMVI) was considered present when a slip of soft tissue was seen extending into the base of a draining vein which appeared unopacified distally ( - Fig. 4A, B).

Nodes were considered metastatic when they were heterogeneous, more than $5 \mathrm{~mm}$ in short axis, with perinodal haziness and irregular contours (-Fig. 4C, D). Distant metastatic work-up was done by studying the rest of the abdomen. In case of hepatic secondaries, resectability analysis was done by vascular and segmental mapping with liver volumetry. CT findings were compared with histopathology (HP) as a reference standard. Pathology reporting was performed by senior pathologists with more than 10 years of experience.

\section{Statistical Analysis}

The sensitivity, specificity, accuracy, positive predictive value, negative predictive value of CT for RC in the PS and post NACRT groups were calculated. The kappa test of agreement was also performed between CT and HP. 

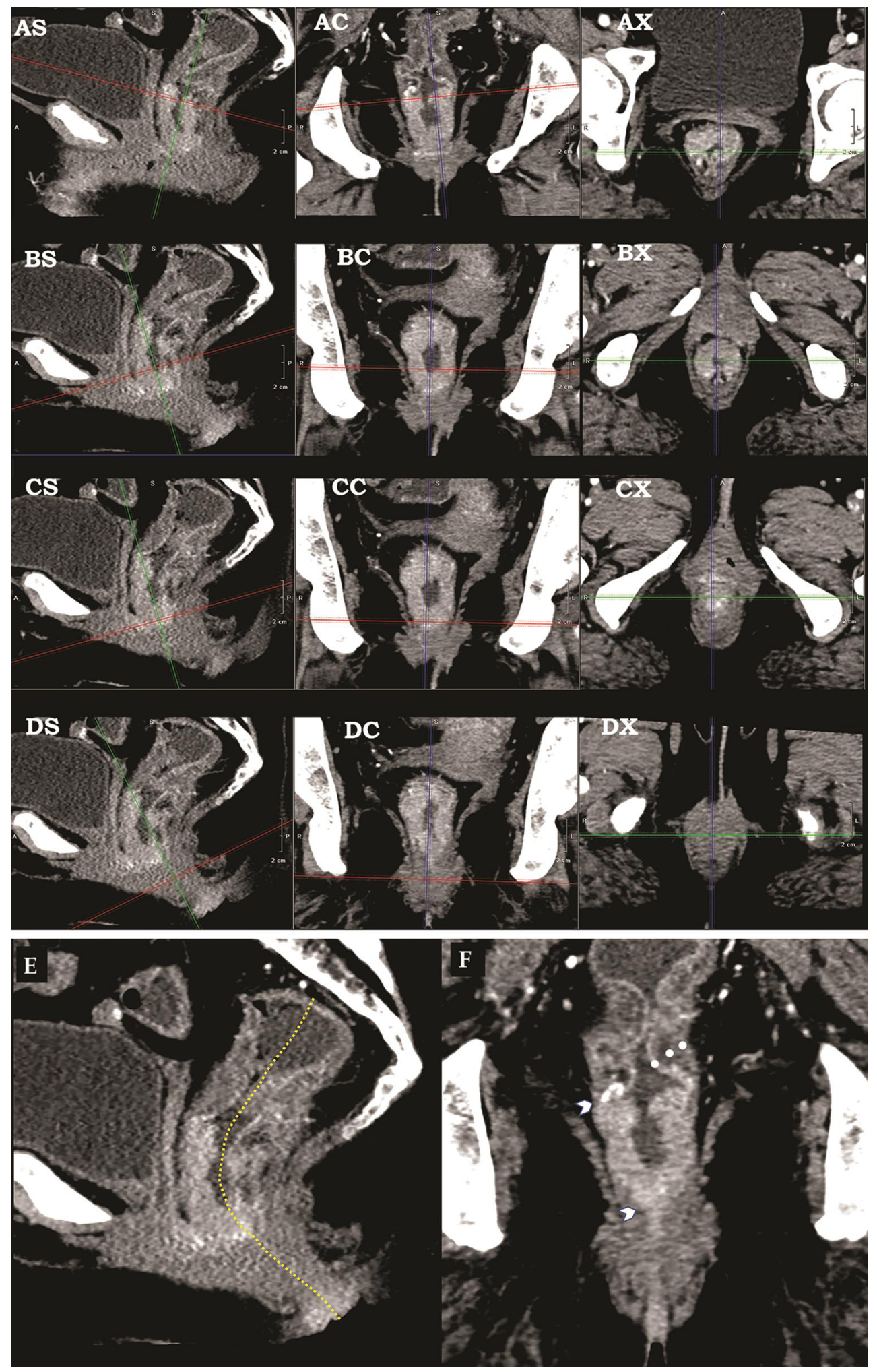

Fig. 1 (A-D) Multiplanar post processing reconstructions through a low rectal cancer in a 54-year-old woman staged at T3c by CT and T3 by HP with R0 resection. A, B, C and D rows represent upper rectum, mid rectum, lower rectum, and anal canal levels, respectively. The letters S, $C$, and $X$ represent sagittal, coronal, and axial sections through the above levels. The cross hair demonstrates how the true orthogonal plane is obtained through the rectum and anal canal. Placing the red line perpendicular to the lumen of the anorectum corrects for the angulation in the anteroposterior plane. Placing the blue line parallel to the length of the rectum corrects for the right to left obliquity. These maneuvers yield a true axial section at AX, BX, CX, and DX images in the panel. Curvilinear reconstruction in (E) shows the centerline (dotted yellow line) drawn through the lumen of the rectum and anal canal. Panel (F) shows the resultant CLR with the entire length of the rectum and anal canal. The upper and lower limits of the RC are marked by arrowheads. The three white dots represent the layers of the normal rectal wall just cranial to the RC. Medial dot-bright mucosa, middle dot-dark submucosa containing fibrofatty tissue, lateral dot-muscularis. CLR, curvilinear reconstruction; HP, histopathology; RC, rectal cancer. 


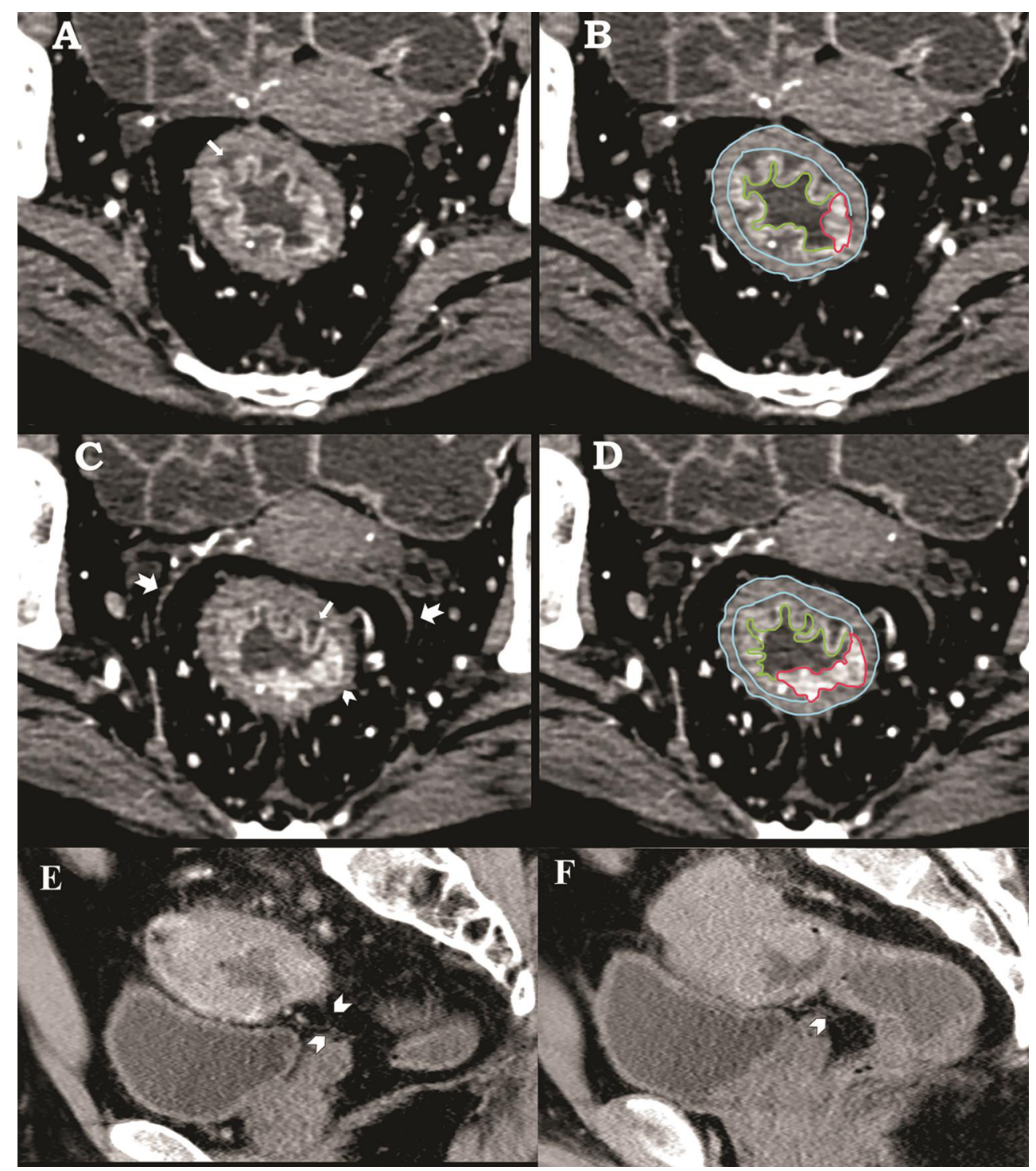

Fig. 2 Panels (A, C) show subsequent axial images in the EVP through the mid rectum of a 63-year-old woman with a well-differentiated RC staged by CT and HP to T2. Panels (B, D) are diagrammatic representations of $\mathbf{A}$ and $\mathbf{C}$, respectively. The mucosa is seen as a bright line drawn green on $B$ and $D$. The submucosa is the dark zone just outside of the mucosa (small arrows in $\mathbf{A}$ and $\mathbf{C}$ ). The muscularis propria is the muscle density layer contained within the two concentric blue lines in B and D. The RC is outlined by red in $\mathbf{B}$ and $\mathbf{D}$ and seen as a brightly enhancing thickening of the mucosa from 3 to 7 o'clock position, growing into the MP but not beyond it (arrowhead in C). The MRF is seen as a thin muscle density line around the mesorectum (notched arrows in C). Panels (E, F) show the anterior peritoneal reflection (arrowheads). EVP, early venous phase; HP, histopathology; MP, muscularis propria; MRF, mesorectal fascia; RC, rectal cancer.

\section{Results}

- Table 1 lists the performance of high-resolution CT for RC staging.

\section{T Stage}

In differentiating stages T2-and-less T2 from T3-T4, the accuracy, sensitivity, specificity, and agreement Kappa score of CT overall were $91 \%, 87 \%, 94 \%$, and 0.8 (excellent), respectively; in the PS group were $89 \%, 76 \%, 94 \%$, and 0.7 (good), respectively (-Table 2); in NACRT group were 97\%, 100\%, 94\%, and 0.9 (excellent), respectively (- Table 3); in low RC group were $89 \%, 97 \%, 94 \%$, and 0.8 (excellent), respectively.

\section{Node-Positive versus Node-Negative Stage}

Node-positive versus node-negative accuracy, sensitivity, specificity, and agreement Kappa score in overall patients were $75 \%, 87 \%, 65 \%$, and 0.5 (fair); in PS group were $72 \%, 93 \%$, $54 \%$, and 0.4 (fair) (- Table 4); in NACRT group were $83 \%, 75 \%$, $88 \%$, and 0.6 (fair) (- Table 5).

\section{Circumferential Resection Margin Status}

Circumferential resection margin (CRM) positivity accuracy, sensitivity, specificity, and agreement Kappa score in overall patients were $91 \%, 81 \%, 94 \%$, and 0.8 (excellent agreement); in PS group were 91\%, 90\%, $88 \%$, and 0.8 (excellent agreement); in NACRT group were $93 \%, 60 \%, 100 \%$, sample 


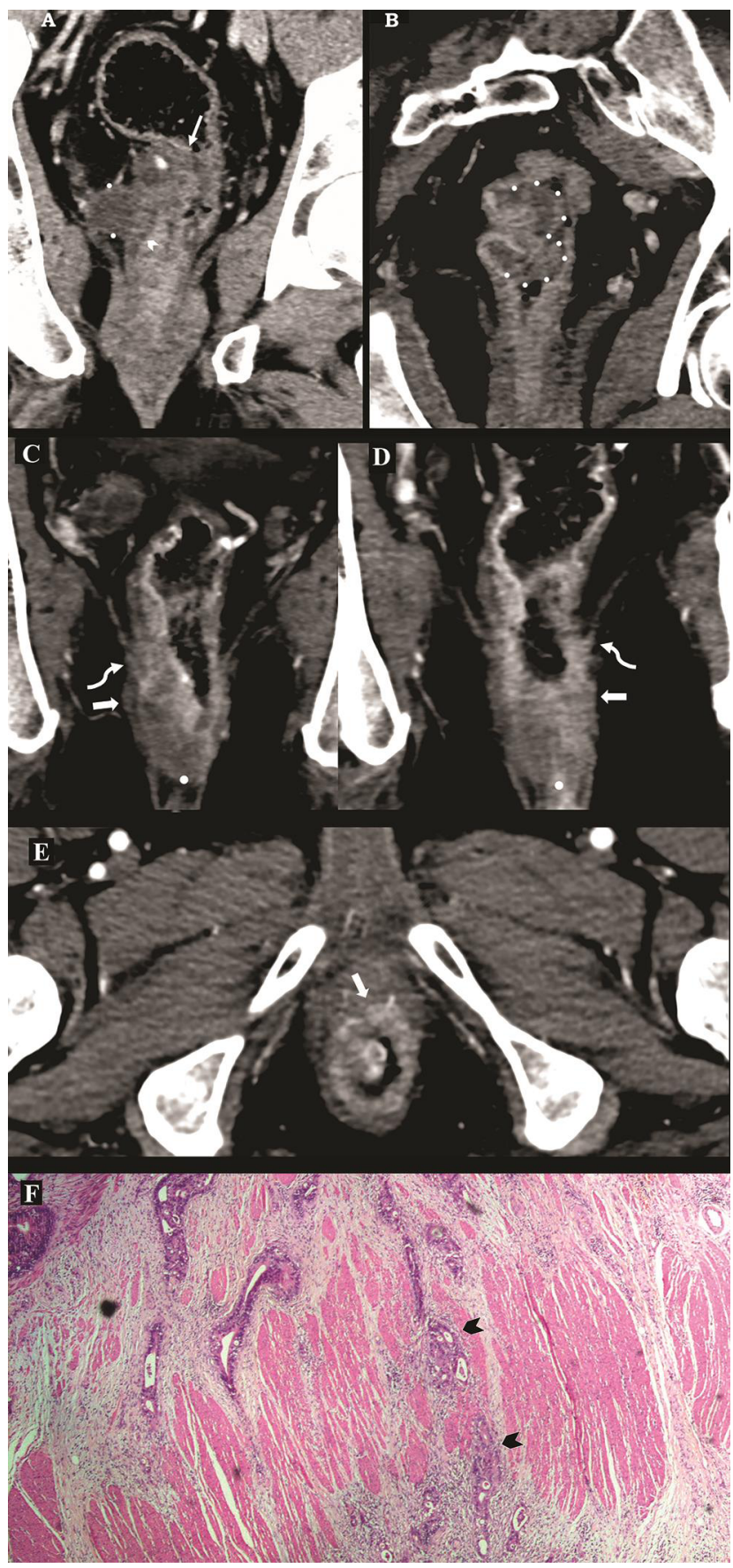

Fig. 3 Panel $(\mathbf{A})$ is a coronal CLR in the EVP of a 43-year-old man with mucinous RC. The tumor is low density compared with the mucosa (marked by the arrow at the upper edge). It grows outward from the right wall (marked by white dots) to involve the levator ani. It also undermines the mucosa and grows beneath it displacing it toward the lumen (arrowhead). Panel (B) shows an oblique coronal planar reconstruction in the EVP of a mucinous low-density polyp marked within dots in a 56-year-old man staged at T1 by both CT and HP. (C, D) Post NACRT RC in a 46-year-old man with partial response staged at yctT4 and matching with HP. CLR in the EVP is rotated to show the inter-sphincteric planes on the right and left, respectively. Curved arrows on both show involvement of the levator ani muscles. Straight arrows reveal sparing of the puborectalis on the right and infiltration on the left. The anal verge is marked by a white dot in each and the tumor is seen extending into the internal sphincter, especially on the left. Panel (E) is axial image in EVP with arrow marking infiltration of the prostate anteriorly. Panel (F) Hematoxylin-eosin 4X HP section reveals the RC (arrowheads) infiltrating into the MP. CLR, curvilinear reconstruction; EVP, early venous phase; HP, histopathology; MP, muscularis propria; NACRT, neoadjuvant chemoradiotherapy; RC, rectal cancer. too small for kappa, respectively. The small sample size also lowered the sensitivity here.

\section{EMVI Status}

EMVI positivity accuracy, sensitivity, specificity, and agreement Kappa score in overall patients were 96\%, 94\%, 96\%, and 0.9 (excellent agreement); in PS group were 98\%, 94\%, $100 \%$, and 0.9 (excellent agreement); in NACRT group were $90 \%, 100 \%, 89 \%$, and sample was too small for kappa, respectively.

\section{Discussion}

\section{Emergent Role of CT for Bowel Wall Imaging and Role of New Post Processing Tools}

With advanced capability to perform submillimeter isotropic sections rapidly in various phases of the contrast, CT has emerged as an accurate modality to assess bowel wall. ${ }^{10,11}$ In our study rectal wall layers are well visualized on CT (-Fig. 5 A-D). In the EVP, the MP and IS do not enhance improving the contrast between these and the RC. In LVP recommended thus far, the MP and IS enhance to become isodense to RC, posing a challenge in assessing depth of invasion (-Fig. 5E, F). Advanced workstation post-processing plays an important role in CT accuracy. With MPR the accuracy of CT staging for RC is higher (87-88\%) as compared with only axial analysis (73-78\%). ${ }^{12,13}$ The rectum is a curvilinear structure. Reconstructions perpendicular to the long axis of the tumor are essential for accurate T-staging. This is feasible easily at $\mathrm{CT}$ oblique postprocessing.

CLR is used for detecting subtle wall abnormalities in the biliary tree and the complex periampullary region. ${ }^{7} \mathrm{CLR}$ allows for display of a hollow viscus on one 2D image and is the mainstay of coronary CT angiograms. ${ }^{14}$ We use this tool with rotation of CLR along the centerline through the lumen of anorectum giving a good view of the wall and relations. Low RC can be aligned with the entire length of the anorectum in one $2 \mathrm{D}$ image giving a straightened-out panoramic view (-Fig. 6).

\section{T Stage and CRM Involvement}

- Table 6 provides a comparison of our study with various studies and meta-analysis on the accuracy of RC staging by CT, MRI, and ERUS. Li et $\mathrm{al}^{4}$ in their meta-analysis of 89 studies found CT (sensitivity $88 \%$, specificity $79 \%$ ) to be equal to MRI (sensitivity $89 \%$, specificity $76 \%$ ) for T staging. Al-Sukhni et al in their meta-analysis of 21 studies found an $87 \%$ sensitivity and $75 \%$ specificity of MRI for T staging. ${ }^{15}$ Our overall sensitivity of $87 \%$ matched and a specificity of $94 \%$ bettered that of MRI for differentiation of T2-and-less than T2 from T3-T4 RC. In our PS group, three out of $13 \mathrm{~T} 2$ were upstaged to T3a whereas, in two patients an erroneous sampling at HP may have caused a mismatch. T staging of some of the polyps presented difficulty in our study. One polyp (T stage on HP [pT]1) that was large, distended the rectum taking the wall close to the MRF (staged ctT3a) (Fig. 7A, B). Two polyps (pT2) located at acutely angulated segments were staged at ctT1. Polyps on straight segments of the rectum were accurately staged 


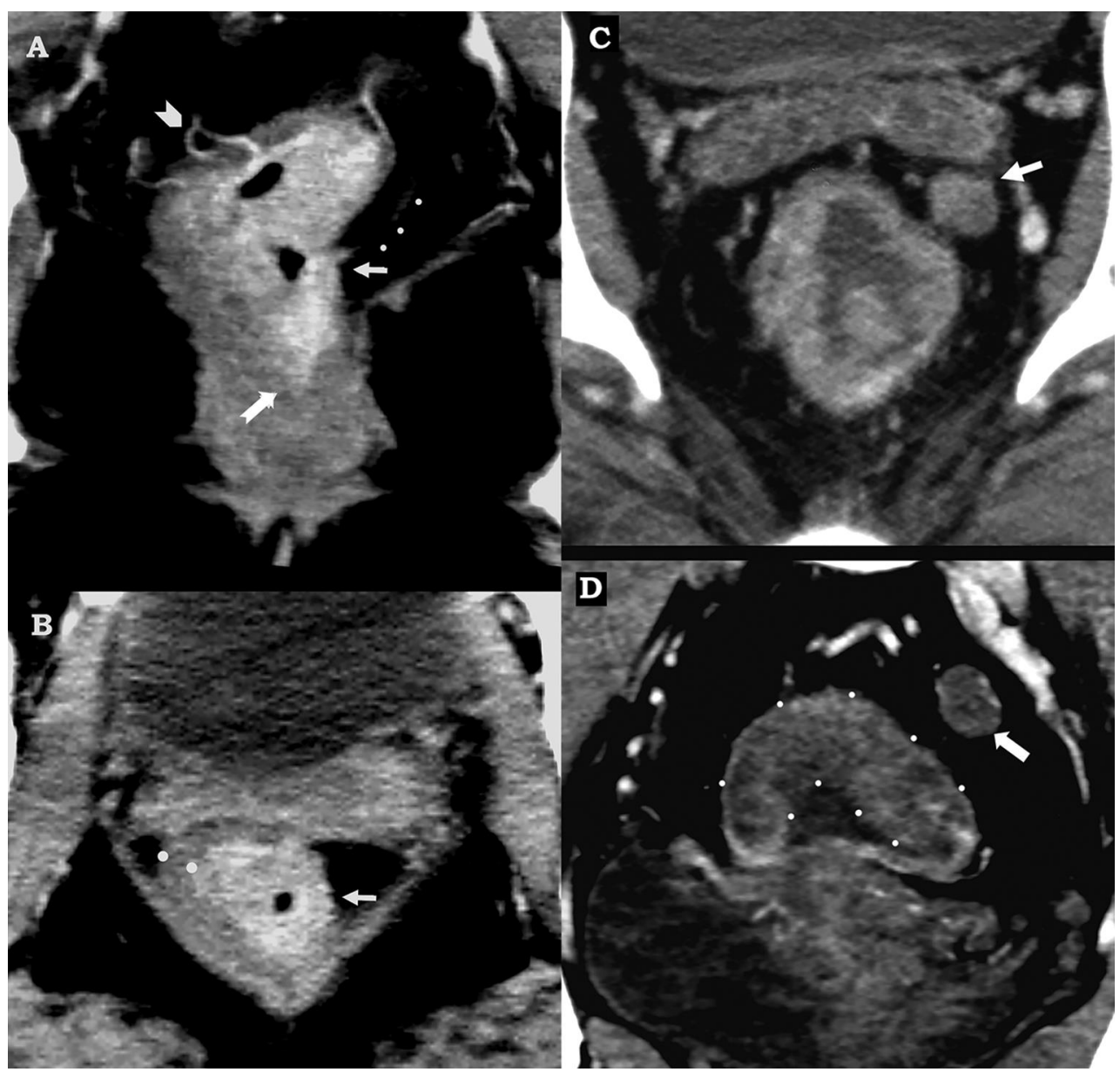

Fig. 4 Coronal oblique planar reconstruction (A) and axial image (B) in the EVP of a 41-year-old woman with a poorly responding low RC post NACRT show a "rose thorn like" slip of enhancing tumor tissue rising into the adjacent vein representing EMVI in panel A (arrow) confirmed on HP. The distal part of the vein is collapsed and marked by small white dots. In comparison the veins without EMVI are well opacified distally (arrowhead in panel A). The leading edge of the enhancing bright tumor infiltrates the low-density internal sphincter (notched arrow in panel A) highlighting the usefulness of the EVP. Panel (B) shows the intact MP on the right within white dots whereas full thickness involvement on the left with minimal extension beyond staged to yctT3a which matched with HP. Axial panel (C) and coronal oblique panel (D) images through the mid rectal RC in late venous phase in two different patients reveal a heterogeneously enhancing metastatic node in panel A (arrow) and poorly enhancing necrotic node (arrow) of a mucinous RC (marked within dots) in panel B. EMVI, extramural vascular invasion; EVP, early venous phase; HP, histopathology; MP, muscularis propria; NACRT, neoadjuvant chemoradiotherapy; RC, rectal cancer.

Table 1 Performance of CT for staging of rectal cancer

\begin{tabular}{|c|c|c|c|c|c|c|c|c|c|c|c|c|c|}
\hline \multirow{2}{*}{$\begin{array}{l}\text { CT staging of } \\
\text { rectal cancer }\end{array}$} & \multicolumn{4}{|c|}{ Overall staging $N=93$} & \multirow{2}{*}{$\begin{array}{l}\text { Low rectal } \\
\text { cancer staging } \\
N=42 \\
<\mathrm{T} 2 \text { vs. } \\
\text { T3-4 }\end{array}$} & \multicolumn{4}{|c|}{ Primary surgery staging $N=64$} & \multicolumn{4}{|c|}{ Post NACRT staging $N=29$} \\
\hline & $\begin{array}{l}\text { T1-2 } \\
\text { vs. } \\
\text { T3-4 }\end{array}$ & $\begin{array}{l}\mathrm{N}+ \\
\text { vs. } \\
\mathrm{N}\end{array}$ & CRM+ & $\begin{array}{l}\text { EMVI } \\
+\end{array}$ & & $\begin{array}{l}\text { T1-2 } \\
\text { vs. } \\
\text { T3-4 }\end{array}$ & $\begin{array}{l}\mathrm{N}+ \\
\text { vs. } \\
\mathrm{N}-\end{array}$ & CRM+ & $\begin{array}{l}\text { EMVI } \\
+\end{array}$ & $\begin{array}{l}<\mathrm{T} 2 \\
\text { vs. } \\
\text { T3- } \\
4\end{array}$ & $\begin{array}{l}\mathrm{N}+ \\
\text { vs. } \\
\mathrm{N}\end{array}$ & CRM+ & $\begin{array}{l}\text { EMVI } \\
+\end{array}$ \\
\hline Sensitivity & 87 & 87 & 81 & 94 & 89 & 76 & 93 & 88 & 94 & 100 & 75 & 60 & 100 \\
\hline Specificity & 94 & 65 & 94 & 96 & 97 & 94 & 54 & 91 & 100 & 94 & 88 & 100 & 89 \\
\hline PPV & 87 & 67 & 82 & 88 & 94 & 81 & 63 & 79 & 100 & 93 & 82 & 100 & 25 \\
\hline NPV & 94 & 87 & 94 & 99 & 94 & 92 & 90 & 95 & 98 & 100 & 83 & 92 & 100 \\
\hline Accuracy & 91 & 75 & 91 & 96 & 94 & 89 & 72 & 90 & 98 & 97 & 83 & 93 & 90 \\
\hline Kappa score & 0.8 & 0.5 & 0.8 & 0.9 & 0.82 & 0.7 & 0.4 & 0.8 & 0.9 & 0.9 & 0.6 & $\mathrm{DI}$ & DI \\
\hline
\end{tabular}

Abbreviations: $\mathrm{CRM}+$, positive circumferential resection margin; DI, data insufficient; EMVI, extramural venous invasion; NACRT, neoadjuvant chemoradiotherapy; NPV, negative predictive value; PPV, positive predictive value.

Note: All values are in percentages. $\mathrm{N}+$ is $\mathrm{N} 1$ or $\mathrm{N} 2$ and $\mathrm{N}$ is N0 stage. 
Table 2 Comparison of CT and histopathological T staging of rectal cancer patients who underwent primary surgery without NACRT

\begin{tabular}{|c|c|c|c|c|c|}
\hline $\begin{array}{l}\text { Primary T staging } \\
\text { Total cases } 64\end{array}$ & ctT1 & ctT2 & ctT3 & ctT4 & \multirow[t]{5}{*}{$\begin{array}{l}\text { Weighted kappa score of } 0.785 \text {. } \\
\text { Good agreement between HP and CT }\end{array}$} \\
\hline pT1(4) & 2 & 1 & 1 & 0 & \\
\hline pT2(13) & 2 & 8 & 3 (T3a) & 0 & \\
\hline pT3 (39) & 0 & 3 (T3a) & 37 & 0 & \\
\hline pT4(7) & 0 & 0 & 0 & 7 & \\
\hline
\end{tabular}

Abbreviation: $\mathrm{HP}$, histopathology.

Note: pT1 to pT4 are histopathological staging. ctT1 to ctT4 are CT staging.

Agreement between HP and CT was good with a weighted Kappa score of 0.785

Table 3 Comparison of CT and histopathological T staging of rectal cancer patients who underwent surgery post NACRT

\begin{tabular}{|l|l|l|l|l|l|l|}
\hline $\begin{array}{l}\text { Post NACRT T staging } \\
\text { Total cases 29 }\end{array}$ & yctT0 & yctT1 & yctT2 & yctT3 & yctT4 & $\begin{array}{l}\text { Weighted kappa score of 0.9. } \\
\text { Very good agreement } \\
\text { between HP and CT }\end{array}$ \\
\hline yрT0(5) & 5 & 0 & 0 & 0 & 0 & \\
\hline урT1(4) & 1 & 2 & 1 & 0 & 0 \\
\hline урT2(5) & 0 & 1 & 3 & $1(T 3 a)$ & 0 \\
\hline урT3(13) & 0 & 0 & 0 & 13 & 0 \\
\hline урT4(2) & 0 & 0 & 0 & 0 & 2 \\
\hline
\end{tabular}

Abbreviations: HP, histopathology; NACRT, neoadjuvant chemoradiotherapy.

Note: ypT1 to ypT4 are histopathological staging. yctT1 to yctT4 are CT staging.

Agreement between HP and CT was very good with a weighted Kappa score of 0.9.

Table 4 Comparison of CT and histopathological N staging of rectal cancer patients who underwent primary surgery without NACRT

\begin{tabular}{|l|l|l|l|l|}
\hline $\begin{array}{l}\text { Primary nodal staging. } \\
\text { Total cases 64 }\end{array}$ & ctN0 & ctN1 & ctN2 & $\begin{array}{l}\text { Weighted kappa score of 0.436. } \\
\text { Moderate agreement between HP and CT }\end{array}$ \\
\cline { 1 - 2 } pN0 (35) & 19 & 10 & 6 & \\
\cline { 1 - 2 } & 2 & 6 & 6 & \\
\hline pN2(15) & 0 & 4 & 11 & \\
\hline
\end{tabular}

Abbreviations: HP, histopathology; NACRT, neoadjuvant chemoradiotherapy.

Note: pN0 to pN2 are histopathological staging. ctN0 to ctN2 are CT staging; Agreement between HP and CT was moderate with a weighted Kappa score of 0.436 .

Table 5 Comparison of CT and histopathological N staging of rectal cancer patients who underwent surgery post NACRT

\begin{tabular}{|l|l|l|l|l|}
\hline $\begin{array}{l}\text { Post NACRT nodal staging } \\
\text { Total cases 29 }\end{array}$ & ctN0 & ctN1 & ctN2 & \multirow{2}{*}{$\begin{array}{l}\text { Weighted kappa score of 0.637. } \\
\text { Good agreement between HP and CT }\end{array}$} \\
\cline { 1 - 4 } pN0(17) & 15 & 1 & 1 \\
\hline pN1(9) & 2 & 7 & 0 \\
\cline { 1 - 3 } pN2(3) & 1 & 0 & 2 & \\
\hline
\end{tabular}

Abbreviations: HP, histopathology; NACRT, neoadjuvant chemoradiotherapy.

Note: pN0 to pN2 are histopathological staging. ctN0 to ctN2 are CT staging; Agreement between HP and CT was good with a weighted Kappa score of 0.637 .

(Fig. 7C-E). Flor et al showed high accuracy of T staging by an apple-core deformity of the wall in stage T3-T4 tumors on CT colonography. ${ }^{16}$ This may hold merit in rectal polyps along acute curves. Wolberink et al, showed a mediocre performance of CT for assessing CRM involvement probably because of the lack of standardization of CT protocol and no MPR. ${ }^{17}$ Whereas, our protocol has yielded a $90 \%$ accuracy for a positive CRM in the PS and 93\% in the NACRT groups.

\section{Low Rectal Cancer T Stage}

Vliegen et al found a reduced CT accuracy in low RC for MRF involvement along the anterior aspect without MPR..$^{18}$ Shida et $\mathrm{al},{ }^{5}$ however, showed that CT accuracy (89\%) surpassed MRI (71\%) in low RC. In our study too, the accuracy of differentiating T2-and-less than T2 from T3-T4 was high at 94\% in low RC (-Fig. 8A-C). Of the 18 cases at T2-and-less, one was over-staged to T3a. Of the 24 pT3-T4, only two were under-staged by CT ( - Table 7 ). This high yield is attributed 

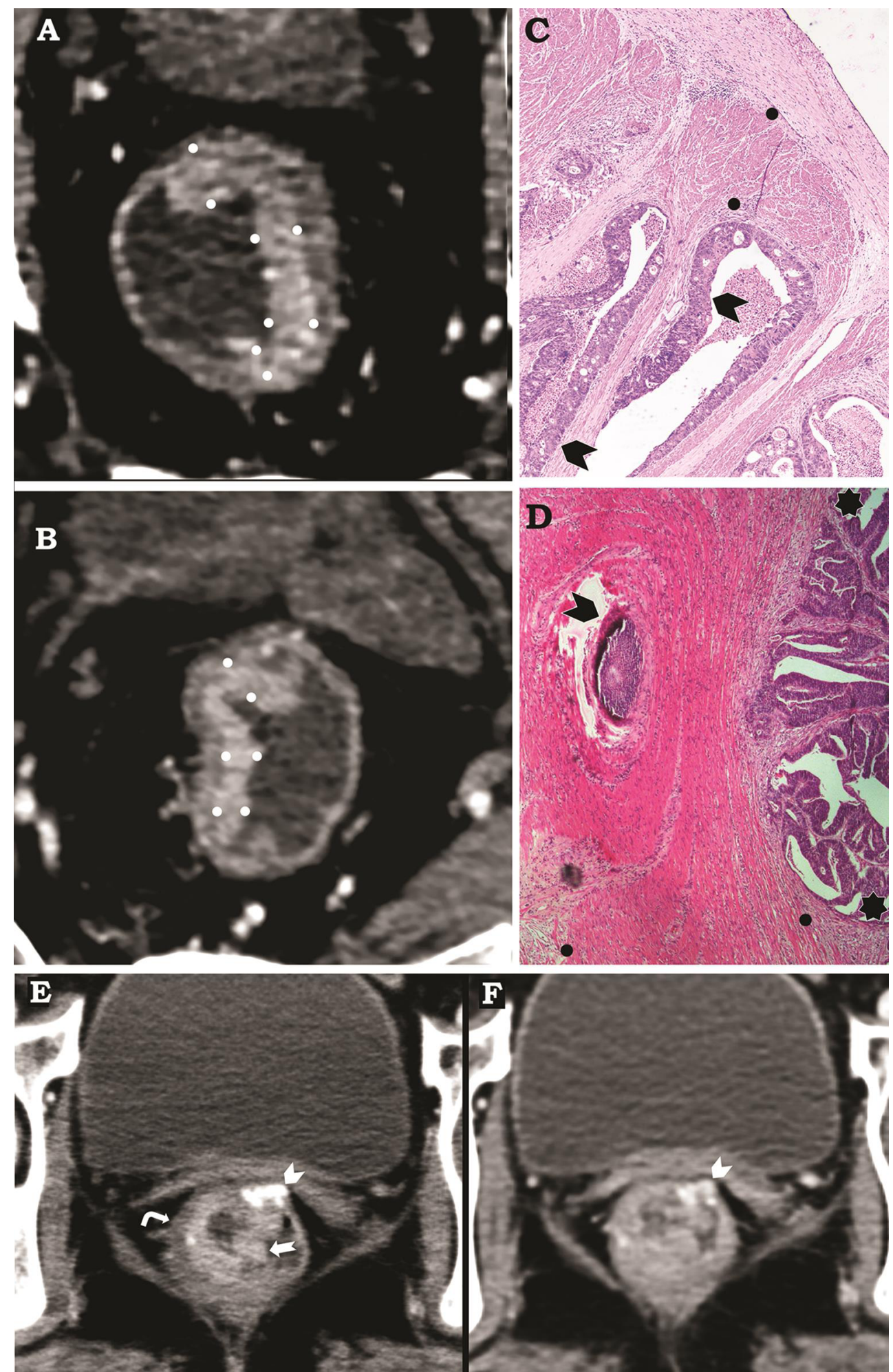

Fig. 5 Axial images in the EVP of Stage T2 RC in two different patients marked within the white dots, on the left wall in panel (A) and right wall in panel (B). RC enhances more than the underlying MP and clearly stands out. Panels (C, D) are corresponding Hematoxylin-eosin 4X HP sections. Arrowheads in C mark the RC and dots mark the MP. In (D) stars mark the RC pushing into the MP (black dots). A tumor nest in the MP is marked by the arrowhead. Axial images in EVP (E) and LVP (F) in a 53-year-old woman with stage T2 low RC demonstrate the usefulness of the EVP with the RC (notched arrow) clearly defined and enhancing brighter than the MP (right angle arrow). Whereas in the LVP the MP enhances to become isodense with the RC and the two cannot be separately seen. The arrowheads mark a large draining vein. EVP, early venous phase; HP, histopathology; LVP, later venous phase; MP, muscularis propria; RC, rectal cancer.

to our high-resolution protocol providing a sharp contrast between the enhancing bright RC that invades the nonenhancing MP and IS (- Fig. 8E, F).

\section{Post NACRT T-Stage}

The accuracy of T-staging was higher in our study for post NACRT (97\%) as compared with PS (89\%). We assessed 


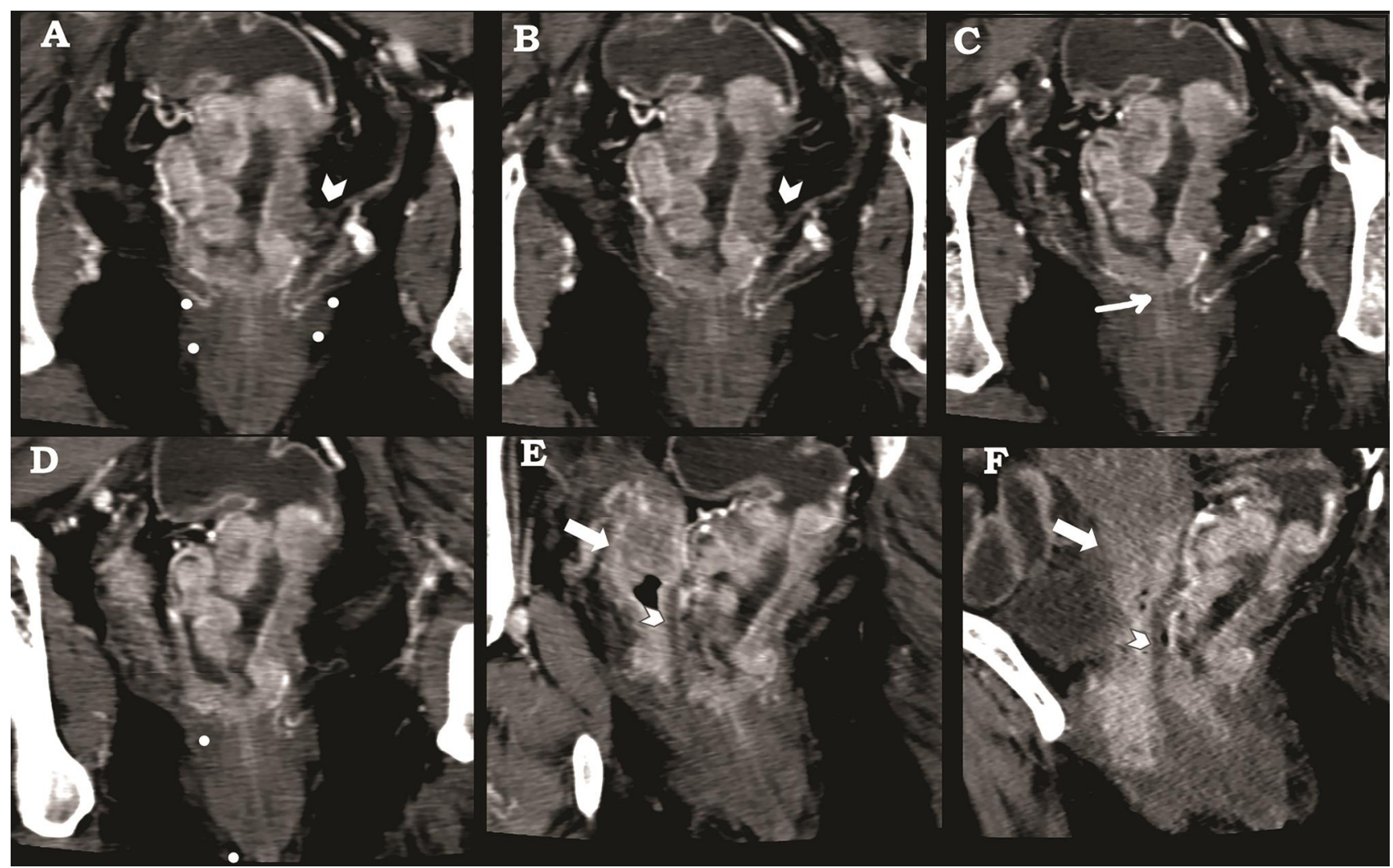

Fig. 6 A series of rotations in a curved reconstruction through the lumen of the rectum shows the low RC (staged at T3 in both CT and HP) and its relation to the MP as well as the anal canal. A clockwise rotation beginning at (A) brings the rectum through left obliques at (A) and (B) into the coronal plane at panel (C). Involvement of the left Levator ani is seen at panels $A$ and $B$ (arrowheads). Further rotation brings right oblique planes at (D) and (E) and nearly sagittal at (F). No involvement of the vagina is seen (arrows in panels $\mathbf{E}$ and $\mathbf{F}$ mark the cervix) with a clear plane between the rectum and the vagina (arrowheads in panels $\mathbf{E}$ and $\mathbf{F}$ ). A sharp margin is seen between the lower edge of the enhancing $\mathrm{RC}$ (curved arrow panel $\mathbf{C}$ ) and the relatively nonenhancing muscular internal sphincter (dots in panel $\mathbf{D}$ ) in the EVP. Dots in (A) mark the puborectalis muscles on either side. The tumor does not cross below this level. EVP, early venous phase; HP, histopathology; MP, muscularis propria; RC, rectal cancer.

viability by tumor enhancement. In complete response, we observed the normal three-layered appearance of the rectum restored post NACRT ( - Fig. 9A, B). In some mucinous tumors the nonviable RC showed fluid density and no enhancement ( - Fig. 9C-H). de Jong et al (46 studies) compared the accuracy of CT, MRI, and ERUS for response post NACRT. CT performed better than MRI with an accuracy for total response of $83 \%$ as compared with MRI of $75 \% .^{19}$ Restaging with diffusion-weighted imaging has shown to improve the mean sensitivity to $83.6 \%$ and specificity to $84.8 \%{ }^{20}$

\section{Nodal Staging}

Nodal staging has low accuracy both on CT and MRI. Size criteria result in only a moderate accuracy. Some authors report lymph nodes with a diameter of $10 \mathrm{~mm}$ or more invariably malignant, ${ }^{3}$ whereas others found $74 \%$ of RC lymph nodes larger than $10 \mathrm{~mm}$ free of cancer and up to $50 \%$ of involved nodes smaller than $5 \mathrm{~mm}$ with $8 \%$ being less than $3 \mathrm{~mm}^{21}$ ( - Fig. 10A, B). RC also causes an antigenic immune challenge to the draining lymph nodes which may result in nodal hyperplasia causing reactive enlargement of regional lymph nodes. ${ }^{21}$ The accuracy for node-positive status in our study was better in the NACRT group with 15 out of 17 node-negative cases correctly identified. The number of total nodes post NACRT were also few in agreement with Koh et al. ${ }^{22}$

\section{Challenges in MRI Local Staging of RC}

Although some studies have reported a high accuracy for MRI, these results have not been widely reproduced. The accuracy of MRI depends on the experience of the radiologist and is subject to significant inter- and intraobserver variability. ${ }^{23} \mathrm{CT}$ is easier to interpret and in one study, examiner's experience was found to have no substantial impact on the reproducibility of CT enterography in the assessment of patients with Crohn's disease. ${ }^{24}$

Peritumoral desmoplastic stranding may give a spiculated appearance beyond the MP overstaging of T2 to T3. ${ }^{3}$ 3T MRI does not add any benefit. ${ }^{25}$ In our study we observed that in the EVP fibrous tissue does not enhance and the desmoplastic spiculations remain hypodense to tumor thus improving differentiation of T2 from T3a (-Figs. 7C, D and 10C-E).

On MRI, appropriate angulation of the axial plane orthogonal to the tumor is inaccurate if the tumor is difficult to identify due to motion artifacts, small size, or intrinsic low contrast between tumor and rectal wall. Redundancy and tortuosity of the rectum can also yield oblique rather than true axial sections with blurring of the MP. ${ }^{23} \mathrm{CT}$ with its dynamic oblique and CLR capabilities can post-process the entire volume 


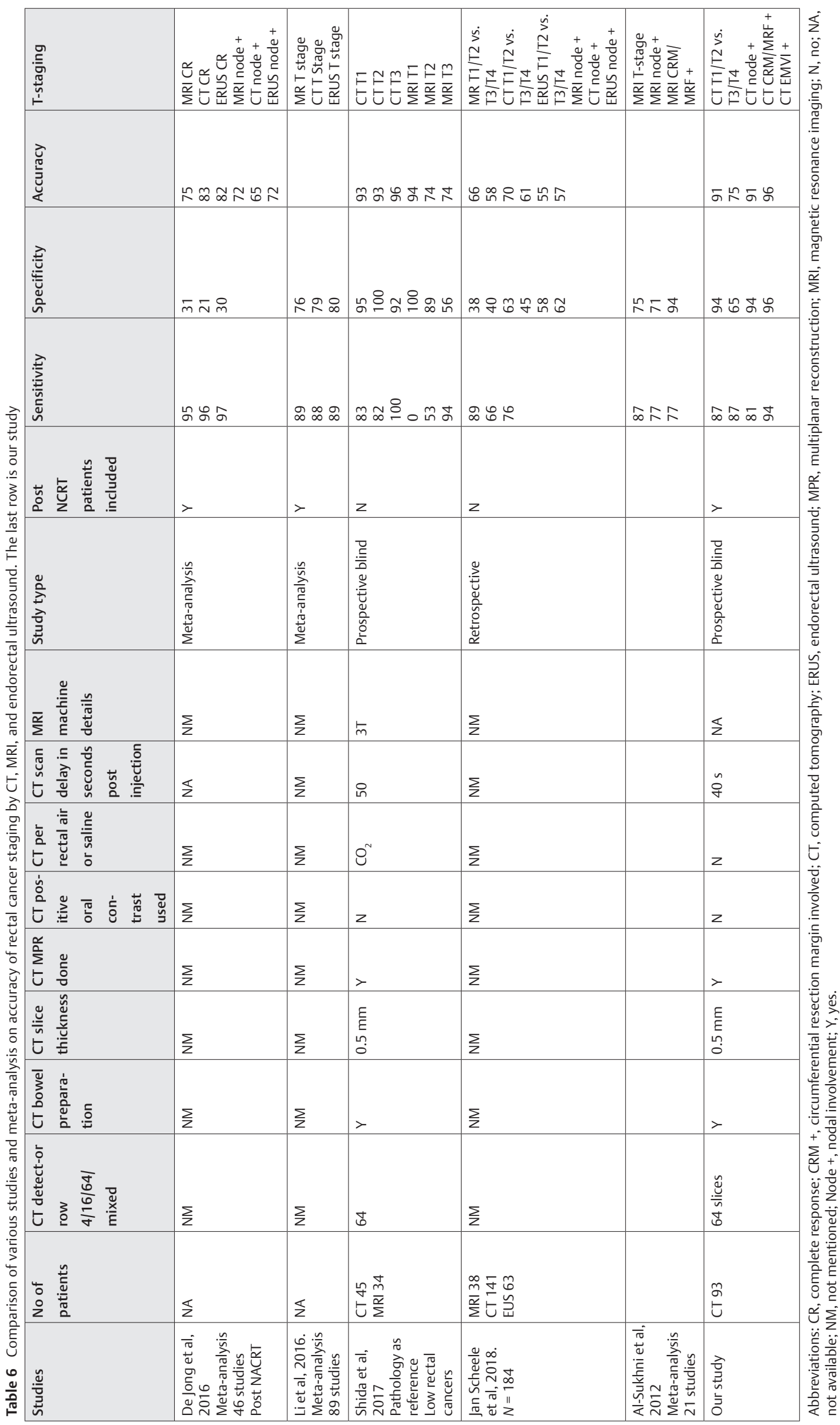



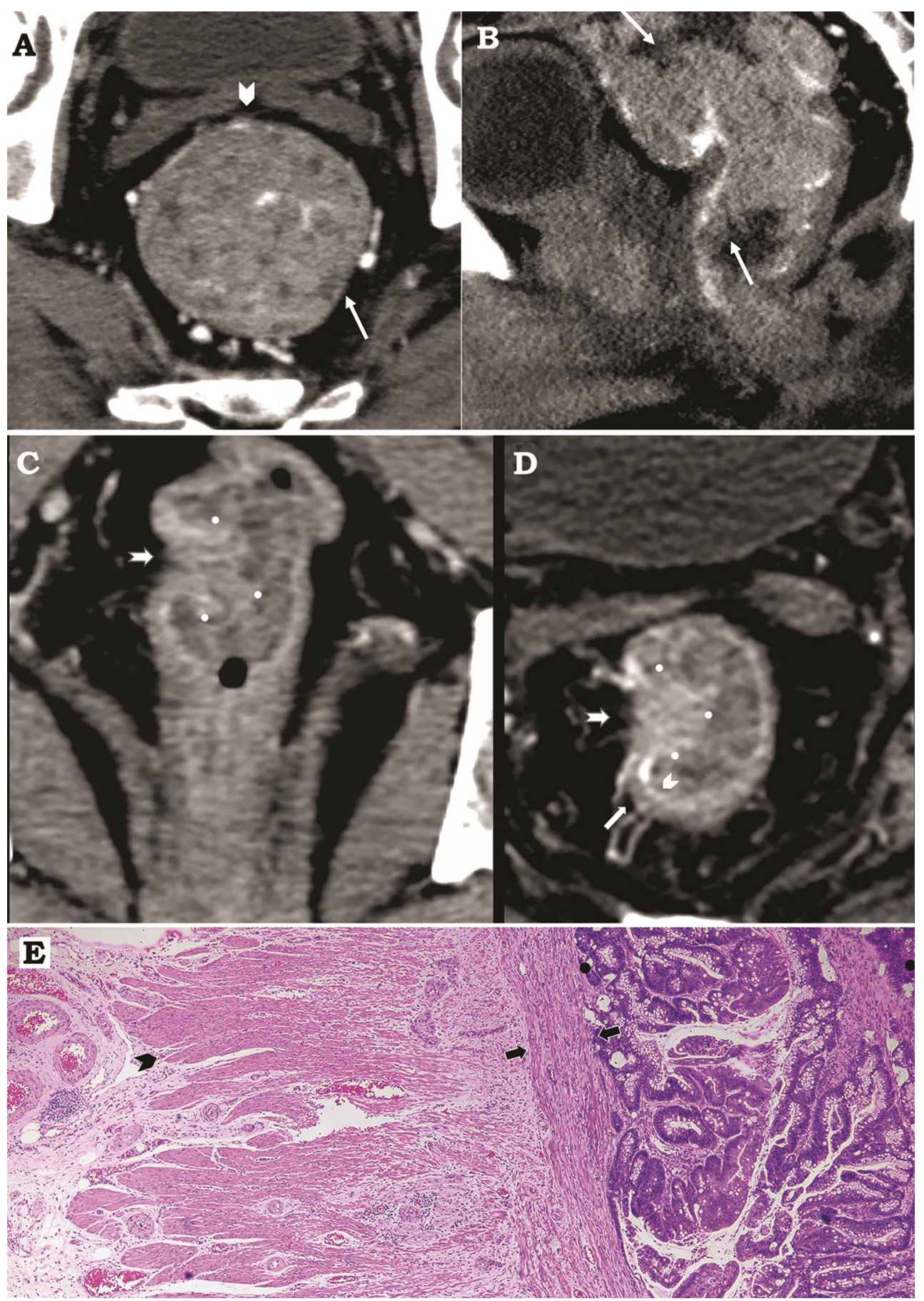

Fig. 7 Panels (A) axial and (B) sagittal images in the EVP of a 59-year-old man with a large rectal polyp arising from the anterior wall marked by arrows on panel $\mathbf{B}$. This was wrongly staged to T3 at CT due to distension of the rectal lumen and bringing the rectal wall close to the MRF anteriorly (arrowhead panel A). The MP is stretched thin and seen only at places (arrow on panel A) making it difficult to assess. Curved coronal reconstruction (C) and axial (D) in the EVP of another 57-year-old man with a stage T2 polyp on both CT and HP arising from the right wall marked within white dots. The mucosa is seen separately (arrowhead in B) from the MP (straight arrow in B). The MP at the base of the polyp is infiltrated and shows similar enhancement as the polyp. Low density spiculations are seen just outside the MP (notched arrow in $\mathbf{A}$ and $\mathbf{B}$ ). These represent desmoplasia and do not enhance in the EVP as much as the RC easing the differentiation of T2 from T3a lesions. Panel (E) Hematoxylin-eosin 4X HP section reveals the RC within black dots pushing into the inner circular layer of the MP marked by arrows. The arrowhead marks the outer edge of the outer longitudinal layer. EVP, early venous phase; $\mathrm{HP}$, histopathology; MP, muscularis propria; MRF, mesorectal fascia; RC, rectal cancer.

dataset with ease, acquiring true axial images and rotating the rectum through a center line along its lumen to see all the walls and its surroundings in the long axis in one 2D image. Lymph nodes along the pelvic sidewall and superior rectal vessels may fall outside the FOV on MRI ${ }^{23}$ but not CT.
Mucinous RCs pose a challenge at restaging by MRI as the mucin persists despite post NACRT. ${ }^{26}$ We compared on CT the pre-NACRT presence of enhancing soft tissues interspersed within mucin for residual enhancement post NACRT which would mean lack of response (-Fig. 9C, D). 


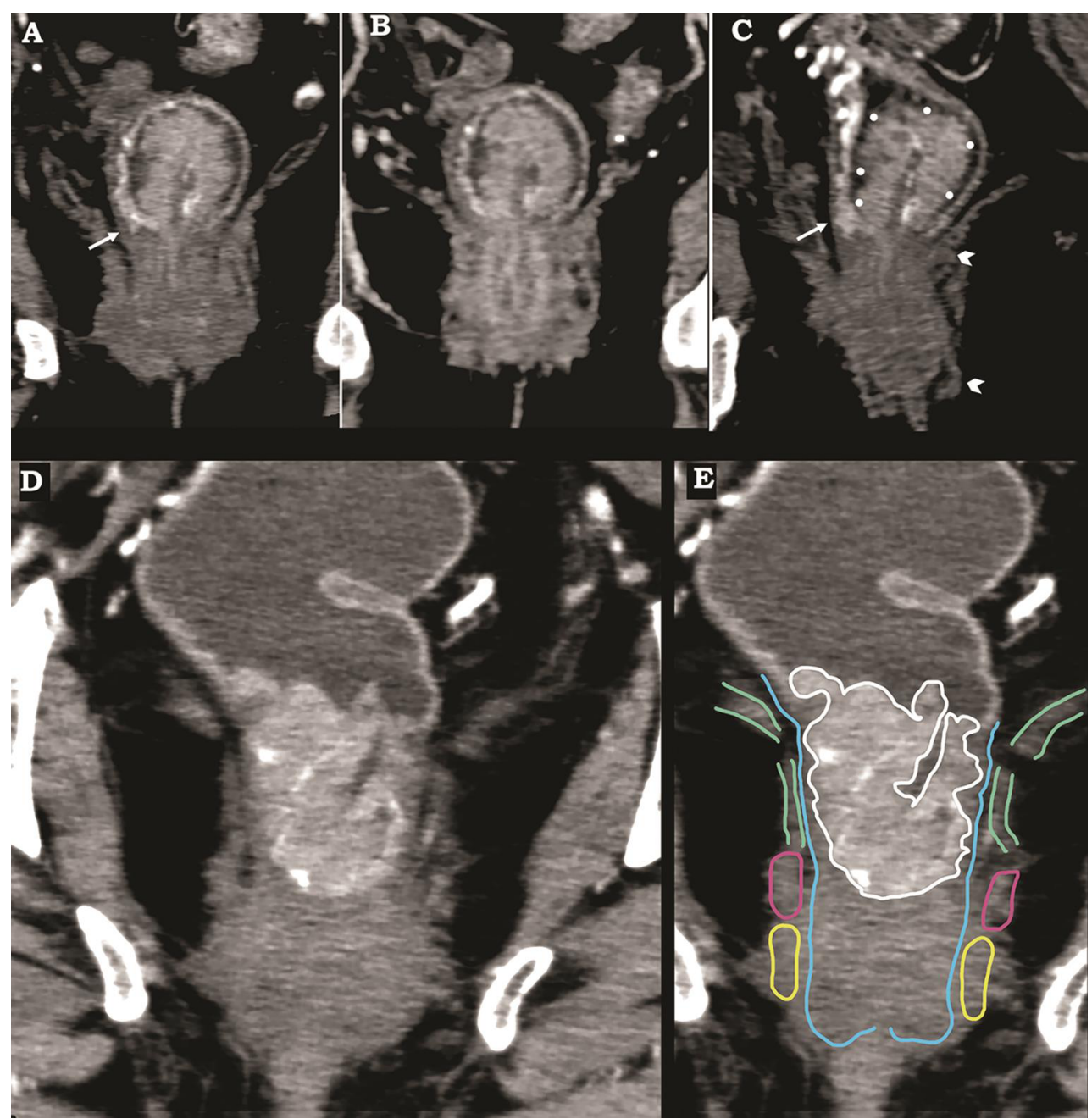

Fig. 8 Low polypoid RC in a 55-year-old woman staged at T2 by both CT and HP. (A) Coronal reconstruction in the EVP. Enhancing tumor tissue is seen along the right side of the polyp at its base infiltrating the MP (arrow). (C) Curvilinear reconstruction in the EVP through the lumen of the anorectum rotated toward the left to open up the right inter-sphincteric plane. The polyp is marked within dots. The infiltration into the MP and not beyond with a clear inter-sphincteric plane (arrow) is better seen. The MP and internal sphincter (arrowheads) do not enhance in this phase sharply demarcating the lower edge of the tumor. In comparison panel (B) (coronal reconstruction in the LVP) shows poor distinction between the RC and MP/internal sphincter as these have begun to enhance in this phase. (D, E) Low RC in a 39-year-old man staged to T2 by both CT and HP. Panel (D) shows a coronal CLR in the EVP. (E) is the diagrammatic representation of D. The RC is outlined in white, Levator ani in green, puborectalis in pink, external sphincter in yellow. and the MP continuing caudally as IS in blue. The IS and MP do not enhance in this EVP with sharp demarcation between them and the RC. CLR, curvilinear reconstruction; EVP, early venous phase; HP, histopathology; LVP, later venous phase; MP, muscularis propria; RC, rectal cancer.

In an era where CT was not of sufficient temporal or spatial resolution to assess the layers of the bowel wall adequately, MRI had an advantage with its superior spatial resolution. It has established itself as the appropriate modality for local staging of RC. Despite this some authors acknowledged that CT could be a good alternative to MRI, when there is a restricted access to MRI, giving the advantage of local and distant staging in a single examination even with a 4 or 16 slice machine. ${ }^{17}$ Scheele et al found that CT had the lowest frequency of therapy relevant over-staging. ${ }^{27}$ Maizlin et al demonstrated substantial agreement between readers in assessment of the tumor, MRF, and lymph nodes on
$\mathrm{CT}^{28}{ }^{28}$ Their study was done on a 4 slice CT scanner, with positive oral contrast medium and no MPR evaluation.

Dar et al concluded that $\mathrm{CT}$ is a reliable radiological tool for local staging of RC with excellent accuracy rates for $\mathrm{T}$ and $\mathrm{N}$ staging. ${ }^{29}$ Today CT perfusion and spectral CT applications provide extraordinary improvements in image quality.

It is known that inventions and radically novel technologies are the result of intentional combinations of two or more existing technologies through a process that involves interplay between experience and knowledge driven by need. ${ }^{30}$ Radiology is not new to this idea of combinatorial evolution. PET-CT and PET-MRI are examples of such. 
Table 7 Comparison of CT and histopathological T staging of overall low rectal cancer patients including those who had primary surgery and those who had surgery post NACRT

\begin{tabular}{|c|c|c|c|c|c|c|}
\hline $\begin{array}{l}\text { Total low rectal cancer } n \\
=42\end{array}$ & ctT0 & ctT1 & ctT2 & ctT3 & ctT4 & \multirow{6}{*}{$\begin{array}{l}\text { Weighted kappa score of } 0.82 \text {. } \\
\text { Very good agreement between } \\
\text { HP and CT }\end{array}$} \\
\hline pT0(4) & 3 & 1 & 0 & 0 & 0 & \\
\hline $\mathrm{pT} 1(6)$ & 1 & 3 & 1 & 1 & 0 & \\
\hline pT2(8) & 0 & 1 & 7 & 0 & 0 & \\
\hline pT3(20) & 0 & 1 & 1 & 18 & 0 & \\
\hline pT4(4) & 0 & 0 & 0 & 0 & 4 & \\
\hline
\end{tabular}

Abbreviations: HP, histopathology; NACRT, neoadjuvant chemoradiotherapy.

Note: PT0 to PT4 are histopathological staging. ctT0 to ctT4 are CT staging. Agreement between HP and CT was very good with a weighted Kappa score of 0.82

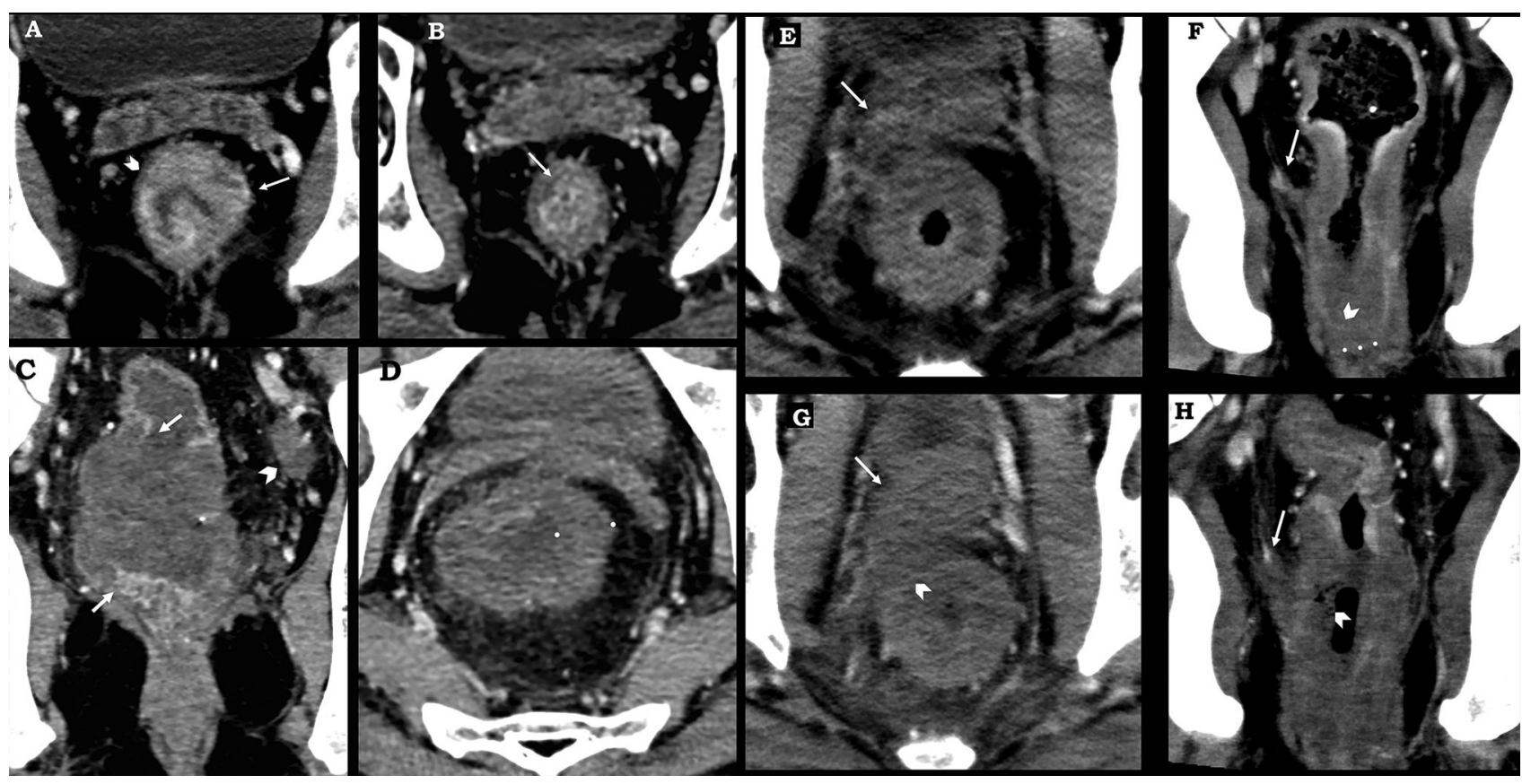

Fig. 9 (A) Pre- and (B) post NACRT low RC EVP axial images in a 32-year-old man. The pre NACRT panel shows the RC sparing the MP at 10 o'clock position (arrowhead). However, at 3 o'clock position the RC infiltrates through the MP (arrow) and extends beyond $5 \mathrm{~mm}$ outward placing the stage at ctT3b. Post NACRT the rectal wall has returned nearly to its normal morphology and the submucosal dark layer is restored (arrow) with mild distortion of the mucosal line. This was staged at yctT1 which matched with HP. (C) Coronal planar reconstruction and (D) axial image through a post NACRT mucinous low RC in a 62-year-old man. There is total necrosis of the RC (dots in panel D) with no discernible enhancement. The normal mucosa and wall at the upper and lower edges of the tumor are marked by arrows. A necrotic node is seen on the left (arrowhead). This was staged at TONO and matched HP. (E) Axial and (F) coronal CLR images in the EVP prior to NACRT and (G, H) post NACRT of a 33-year-old man with signet ring cell adenocarcinoma. (E) shows a ctT4b concentric stenosing type of tumor infiltrating the prostate with an enhancing advancing edge (arrow). Post NACRT (G) shows no enhancement in this region with only low-density necrosis. The rectal wall margin is seen away from the MRF (arrowhead) and this was restaged to yctT3c. HP revealed CRM negative with a clearance of $0.2 \mathrm{~mm}$ and matched that of CT. Panel (F) shows an enhancing EMVI (arrow) pre NACRT. This shows necrosis in post NACRT (H) along with significant necrosis and ulceration (arrowhead) within the tumor. The lower edge of the tumor was seen well below the level of the puborectalis (arrowhead in panel $\mathbf{F}$ ). The anal verge is marked by three dots. The APR specimen revealed tumor at $1.7 \mathrm{~cm}$ from the distal resection margin. APR, abdominoperineal resection; CLR, curvilinear reconstruction; CRM, circumferential resection margin; EVP, early venous phase; HP, histopathology; MP, muscularis propria; MRF, mesorectal fascia; NACRT, neoadjuvant chemoradiotherapy; RC, rectal cancer.

Likewise, CT and MRI can be utilized in a combinatorial manner to yield higher accuracy and better outcomes for RC patients.

Our study proves that high-resolution, contrast-enhanced, thin section CT performed with a small FOV in the EVP yields high contrast between the $\mathrm{RC}$ and the various layers of rectal wall and is an accurate tool for local staging of RC.

The limitation of our study is the small number of patients especially in the post NACRT group. Further, large volume studies are required to validate our findings. 


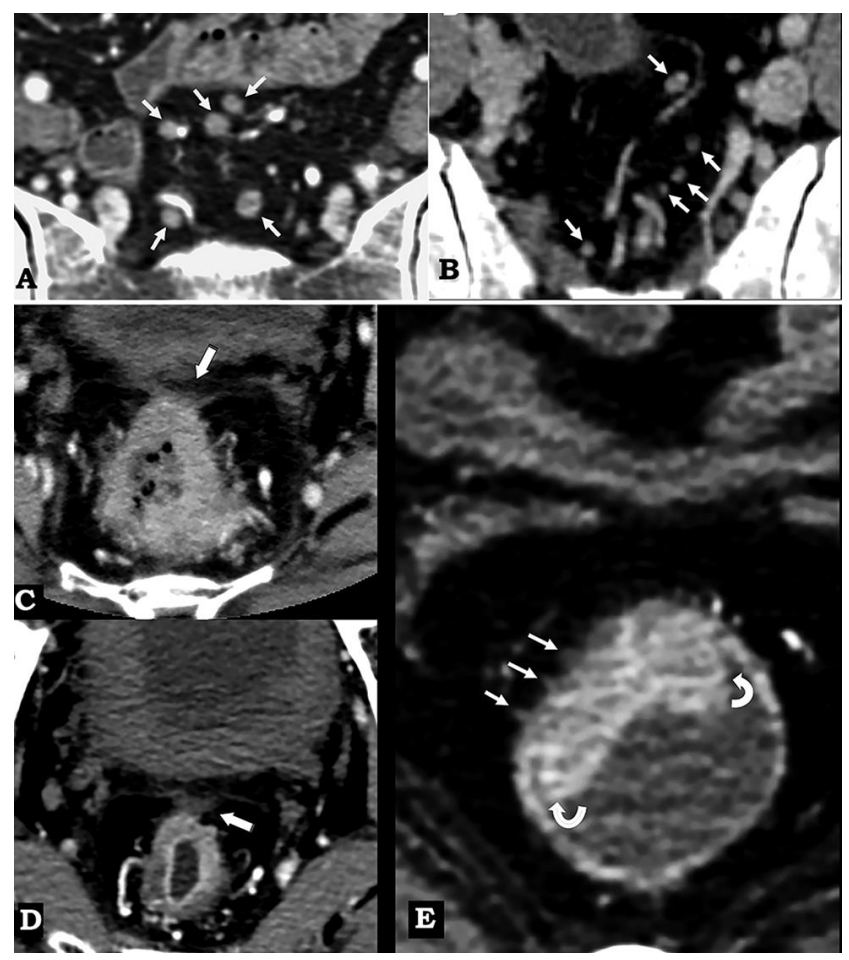

Fig. 10 Axial images in the LVP of two patients. Panel (A) shows nodes larger than $5 \mathrm{~mm}$ considered positive on CT (arrows). All of these were negative on HP with an NO status. Panel (B) shows nodes less than $5 \mathrm{~mm}$ considered negative on CT (arrows). All of these were positive on HP. (C) Pre and (D) post NACRT axial images in the EVP of a 67-year-old man. A bulky concentric RC staged at T3c on CT is seen in (C) with involvement of the MRF anteriorly (arrow) as well as posteriorly. Post NACRT the anterior tumor margin has dropped away from the MRF leaving behind a nonenhancing tissue representing fibrosis. Residual enhancing tumor persists from 10 to 4 o'clock in the MP and minimally beyond it (yctT3a corelating with HP). In the rest of the posterior wall the MP has returned to the normal low enhancement suggesting absence of viable tumor here. Panel (E) is an axial section in the EVP of another 59-year-old man with a mid RC accurately staged at $\mathrm{T} 2$ by $\mathrm{CT}$. Desmoplastic low-density spicules (straight arrows) are clearly differentiable from the enhancing tumor confined to the MP. Curved arrows mark the overhanging lateral margins of the RC in the lumen. EVP, early venous phase; HP, histopathology; LVP, later venous phase; MP, muscularis propria; MRF, mesorectal fascia; NACRT, neoadjuvant chemoradiotherapy; RC, rectal cancer.

\section{Funding}

None.

\section{Conflict of Interest \\ None declared.}

\section{References}

1 Raman SP, Chen Y, Fishman EK. Evolution of imaging in rectal cancer: multimodality imaging with MDCT, MRI, and PET. J Gastrointest Oncol 2015;6(2):172-184

2 Mathur P, Smith JJ, Ramsey C, et al. Comparison of CT and MRI in the pre-operative staging of rectal adenocarcinoma and prediction of circumferential resection margin involvement by MRI. Colorectal Dis 2003;5(5):396-401

3 Beets-Tan RG, Beets GL. Local staging of rectal cancer: a review of imaging. J Magn Reson Imaging 2011;33(5):1012-1019
4 Li XT, Zhang XY, Sun YS, Tang L, Cao K. Evaluating rectal tumor staging with magnetic resonance imaging, computed tomography, and endoluminal ultrasound: a meta-analysis. Medicine (Baltimore) 2016;95(44):e5333

5 Shida D, Iinuma G, Komono A, et al. Preoperative T staging using CT colonography with multiplanar reconstruction for very low rectal cancer. BMC Cancer 2017;17(1):764

6 Goh V, Glynne-Jones R. Perfusion CT imaging of colorectal cancer. Br J Radiol 2014;87(1034):20130811

7 Raman SP, Fishman EK. Abnormalities of the distal common bile duct and ampulla: diagnostic approach and differential diagnosis using multiplanar reformations and 3D imaging. AJR Am J Roentgenol 2014;203(1):17-28

8 Zhu H, Zhang L, Wang Y, et al. Improved image quality and diagnostic potential using ultra-high-resolution computed tomography of the lung with small scan FOV: a prospective study. PLoS One 2017;12(2):e0172688

9 Nishiharu T, Yamashita Y, Ogata I, Sumi S, Mitsuzaki K, Takahashi M. Spiral CT of the pancreas. The value of small field-of-view targeted reconstruction. Acta Radiol 1998;39(1):60-63

10 Sheedy SP, Kolbe AB, Fletcher JG, Fidler JL. Computed tomography enterography. Radiol Clin North Am 2018;56(5):649-670

11 Sharma R, Madhusudhan KS, Ahuja V. Intestinal tuberculosis versus Crohn's disease: clinical and radiological recommendations. Indian J Radiol Imaging 2016;26(2):161-172

12 Sinha R, Verma R, Rajesh A, Richards CJ. Diagnostic value of multidetector row CT in rectal cancer staging: comparison of multiplanar and axial images with histopathology. Clin Radiol 2006;61(11):924-931

13 Ippolito D, Drago SG, Franzesi CT, Fior D, Sironi S. Rectal cancer staging: multidetector-row computed tomography diagnostic accuracy in assessment of mesorectal fascia invasion. World J Gastroenterol 2016;22(20):4891-4900

14 Karlo CA, Leschka S, Stolzmann P, Glaser-Gallion N, Wildermuth S, Alkadhi H. A systematic approach for analysis, interpretation, and reporting of coronary CTA studies. Insights Imaging 2012;3(3):215-228

15 Al-Sukhni E, Milot L, Fruitman M, et al. Diagnostic accuracy of MRI for assessment of T category, lymph node metastases, and circumferential resection margin involvement in patients with rectal cancer: a systematic review and meta-analysis. Ann Surg Oncol 2012;19(7):2212-2223

16 Flor N, Mezzanzanica M, Rigamonti P, et al. Contrast-enhanced computed tomography colonography in preoperative distinction between T1-T2 and T3-T4 staging of colon cancer. Acad Radiol 2013;20(5):590-595

17 Wolberink SV, Beets-Tan RG, de Haas-Kock DF, van de Jagt EJ, Span MM, Wiggers T. Multislice CT as a primary screening tool for the prediction of an involved mesorectal fascia and distant metastases in primary rectal cancer: a multicenter study. Dis Colon Rectum 2009;52(5):928-934

18 Vliegen R, Dresen R, Beets G, et al. The accuracy of multi-detector row CT for the assessment of tumor invasion of the mesorectal fascia in primary rectal cancer. Abdom Imaging 2008;33(5):604-610

19 de Jong EA, ten Berge JC, Dwarkasing RS, Rijkers AP, van Eijck $\mathrm{CH}$. The accuracy of MRI, endorectal ultrasonography, and computed tomography in predicting the response of locally advanced rectal cancer after preoperative therapy: a metaanalysis. Surgery 2016;159(3):688-699

20 van der Paardt MP, Zagers MB, Beets-Tan RG, Stoker J, Bipat S. Patients who undergo preoperative chemoradiotherapy for locally advanced rectal cancer restaged by using diagnostic MR imaging: a systematic review and meta-analysis. Radiology 2013;269(1):101-112

21 Rössler O, Betge J, Harbaum L, Mrak K, Tschmelitsch J, Langner C. Tumor size, tumor location, and antitumor 
inflammatory response are associated with lymph node size in colorectal cancer patients. Mod Pathol 2017;30(6):897-904

22 Koh DM, Chau I, Tait D, Wotherspoon A, Cunningham D, Brown G. Evaluating mesorectal lymph nodes in rectal cancer before and after neoadjuvant chemoradiation using thin-section T2-weighted magnetic resonance imaging. Int J Radiat Oncol Biol Phys 2008;71(2):456-461

23 Kaur $\mathrm{H}$, Choi $\mathrm{H}$, You YN, et al. MR imaging for preoperative evaluation of primary rectal cancer: practical considerations. Radiographics 2012;32(2):389-409

24 Burlin S, Favaro LR, Bretas EA, et al. Using computed tomography enterography to evaluate patients with Crohn's disease: what impact does examiner experience have on the reproducibility of the method? Radiol Bras 2017;50(1):13-18

25 Maas M, Lambregts DM, Lahaye MJ, et al. T-staging of rectal cancer: accuracy of 3.0 Tesla MRI compared with 1.5 Tesla. Abdom Imaging 2012;37(3):475-481
26 Wnorowski AM, Menias CO, Pickhardt PJ, Kim DH, Hara AK, Lubner MG. Mucin-containing rectal carcinomas: overview of unique clinical and imaging features. AJR Am J Roentgenol 2019;213(1):26-34

27 Scheele J, Schmidt SA, Tenzer S, Henne-Bruns D, Kornmann M. Overstaging: a challenge in rectal cancer treatment. Visc Med 2018;34(4):301-306

28 Maizlin ZV, Brown JA, So G, et al. Can CT replace MRI in preoperative assessment of the circumferential resection margin in rectal cancer? Dis Colon Rectum 2010;53(3):308-314

29 Dar RA, Chowdri NA, Parray FQ Shaheen F, Wani SH, Mushtaque M. Pre-operative staging of rectal cancer using multi-detector row computed tomography with multiplanar reformations: single center experience. Indian J Cancer 2014;51(2):170-175

30 Brian Arthur W, The mechanisms of evolution. In: The Nature of Technology. What It Is and How It Evolves. 1st ed. London, Great Britain: Penguin books; 2010 167- 189 